\title{
Dark Triad and Sadism: Effects on Perpetration of Intimate Partner Violence
}

IPV is a form of violence against women, or a type of gender-based violence, which includes physical, sexual, or psychological violence perpetrated by a current or former intimate partner (United Nations, 1993). Regional estimates of IPV prevalence over lifetime vary widely, ranging from a low prevalence of $16.3 \%$ (East Asia) to a high prevalence of $65.6 \%$ (Central Sub-Saharan Africa). It is estimated that the prevalence of IPV over lifetime, in Western Europe and North America, is approximately $20 \%$ (Devries et al., 2013). The literature has reported that personality traits such as Machiavellianism, Psychopathy, Narcissism and Sadism influence the practice of IPV.

\section{AlM}

Which instruments are most commonly used in the dark triad and in sadism?
How do specific personality traits influence the

practice of IPV?

\section{METHOD}

Search expression used:

TX (psychopathy OR narcissism OR machiavellianism OR sadism) AND TI (domestic violence or domestic abuse or intimate partner violence or partner violence or spousal abuse) NOT TI psychopatho*.

\section{Inclusion Criteria}

(a) Empirical study;

(b) Sample older than 18 years;

(c) IPV studies

(d) One of the personality traits.

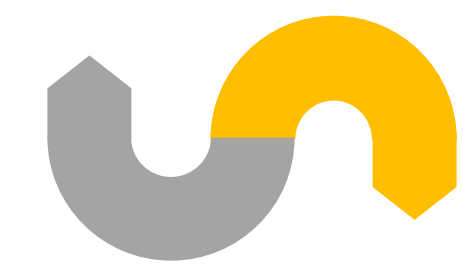

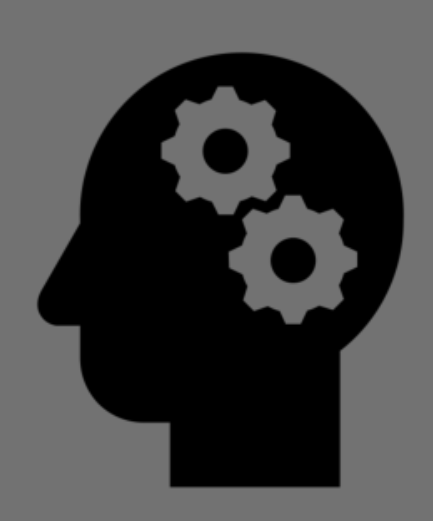

\section{INTRODUCTION}

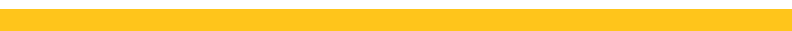

\section{MACHIAVELLIANISM}

- Machiavellianism subscale

- Short Dark Triad

\section{PSYCHOPATHY (4)}

- Psychopathic Personality Inventory

- Self-Report Psychopathy Scale

- Psychopathy Checklist

- Levenson Self-Report Psychopathy Scales

\section{NARCISSISM}

- Narcissistic Personality Inventory

- Short Dark Triad

\section{SADISM}

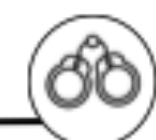

- Sadistic Impulse Scale

- Short Dark Triad

\section{CONCLUSIONS}

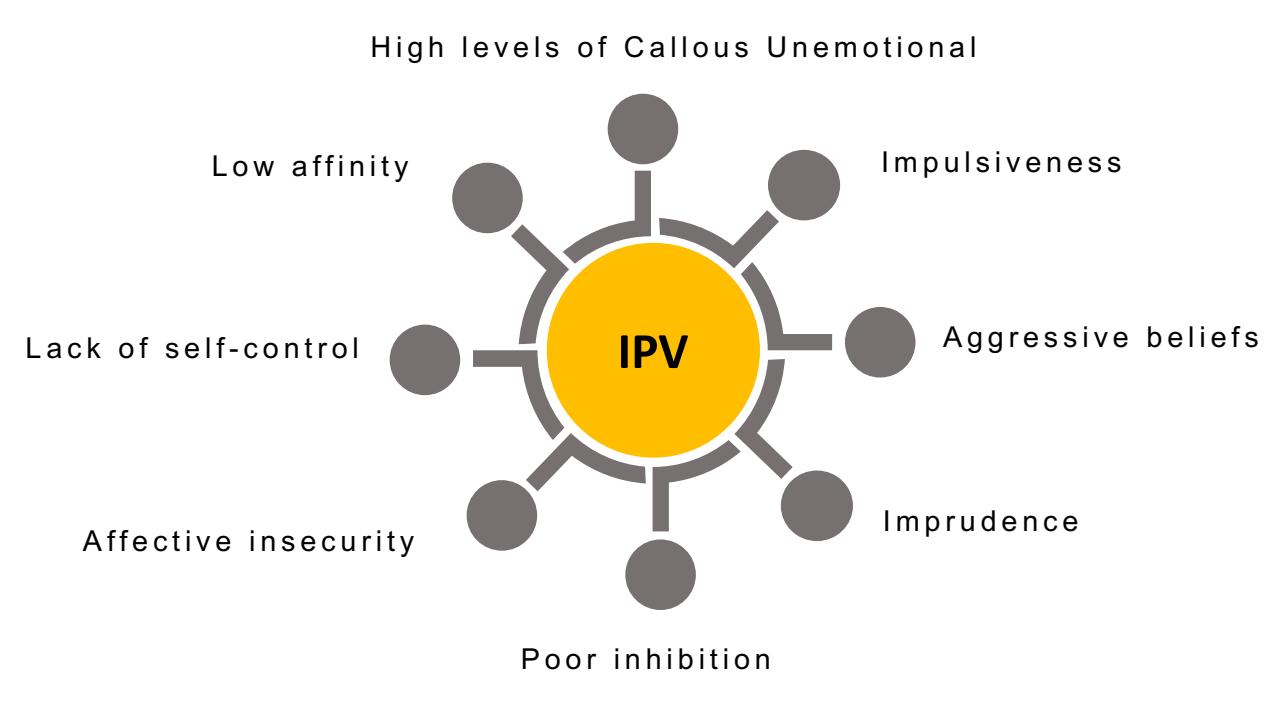

(a) Review studies;

(b) Theoretical essays;

(c) Studies limited to children/adolescents;

(d) Case studies.
IDENTIFICATION SCREENING ELIGIBILITY INCLUDED

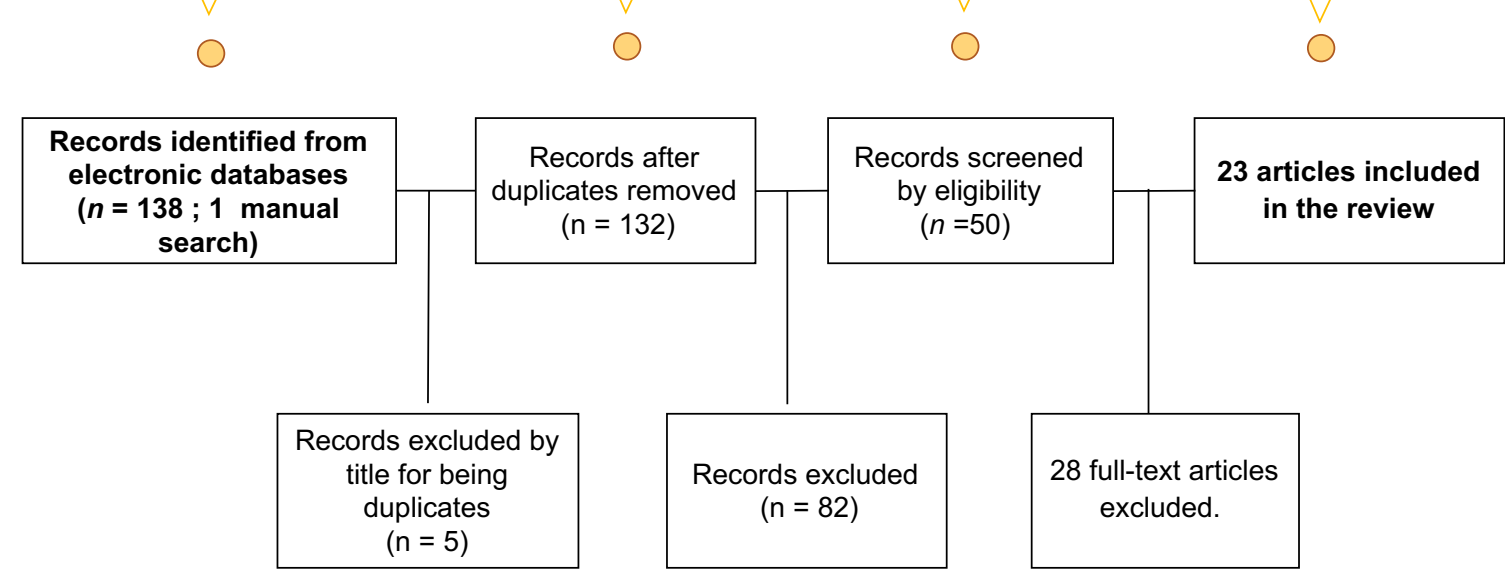

